\title{
ON THE ODD-EVEN HOPSCOTCH SCHEME FOR THE NUMERICAL INTEGRATION OF TIME-DEPENDENT PARTIAL DIFFERENTIAL EQUATIONS
}

\author{
J.H.M. TEN THIJE BOONKKAMP and J.G. VERWER \\ Centre for Mathematics and Computer Science, 1098 SJ Amsterdam, The Netherlands
}

\begin{abstract}
This paper is devoted to the odd-even hopscotch scheme for the numerical integration of time-dependent partial differential equations. Attention is focussed on two aspects. Firstly, via the equivalence to the combined leapfrog-Du Fort-Frankel method we derive the explicit expression of the critical time step for von Neumann stability for a class of multi-dimensional convection-diffusion equations. This expression can be derived directly by applying a useful stability theorem due to Hindmarsh, Gresho and Griffiths [9]. The interesting thing on the critical time step is that it is independent of the diffusion parameter and yet smaller than the critical time step for zero diffusion, but only in the multi-dimensional case. This curious phenomenon does not occur for the one-dimensional problem. Secondly, we consider the drawback of the Du Fort-Frankel accuracy deficiency of the hopscotch scheme. To overcome this deficiency we discuss global Richardson extrapolation in time. This simple device can always be used without reducing feasibility. Numerical examples are given to illustrate the outcome of the extrapolation.
\end{abstract}

\section{Introduction}

The subject of this paper is the odd-even hopscotch (OEH) method for the numerical integration in time of time-dependent partial differential equations (PDEs) (Gordon [2], Gourlay [3-5]). Attention is focussed on two aspects. First we consider the $d$-space dimensional convection-diffusion equation

$$
u_{t}+(q \cdot \nabla) u=\epsilon \Delta u, \quad t>0, \quad x \in \mathbb{R}^{d},
$$

where $u(x, t) \in \mathbb{R}$ represents the convected and diffused variable, the vector $q=\left(q_{1}, \ldots, q_{d}\right)$ the (constant) velocity, and $\epsilon>0$ a diffusion parameter. When combined with simple central differences the $\mathrm{OEH}$ method shows an equivalence to the combined leapfrog-Du Fort-Frankel method. Via this equivalence we derive the explicit expression of the critical time step for von Neumann stability for problem (1.1). This expression is easily found by applying a useful theorem due to Hindmarsh, Gresho and Griffiths [9]. This theorem plays an important role in their stability analysis of the forward Euler-central difference scheme.

The interesting thing on the critical time step is that it is independent of $\epsilon$ whereas it is smaller than the critical time step for zero diffusion, but only in the multi-dimensional case. We wish to remark that this pathological behaviour of the leapfrog-Du Fort-Frankel method has been observed earlier (see [13] and the references therein). However, to the best of our knowledge, the explicit expression of the critical time step is new.

An immediate consequence of this pathological behaviour is that adding artificial diffusion to the $\mathrm{OEH}$ central difference scheme may render the process unstable. This observation is in clear 
contrast to the common practice which teaches us that introducing artificial diffusion has a stabilizing effect. We note that this remark does not contradict the findings of Gourlay and Morris [6] in their investigation of the OEH scheme for nonlinear shock calculations as they restrict their attention to the one-dimensional case.

The second aspect of the OEH method considered in this paper is the drawback we refer to as the Du Fort-Frankel (DFF) accuracy deficiency. The consequence of the DFF deficiency is that convergence takes place for a smaller set of rules for refinement of the time-space mesh than allowed by stability [12]. To overcome this deficiency we discuss global Richardson extrapolation in time. This simple device can be placed on top of any $\mathrm{OEH}$ implementation without reducing feasibility. We present two examples to illustrate the technique.

\section{The $\mathrm{OEH}$ method}

In this section we briefly recall the $\mathrm{OEH}$ method which was first suggested by Gordon [2]. For an extensive discussion we refer to the work of Gourlay who invented the name hopscotch and made a thorough study of various techniques. Here we adopt his formulation.

Let the general form $u_{t}=L u$ represent an evolutionary problem for a system of PDEs in $d$-space dimensions. Boundary conditions will not be specified here as we do not discuss their influence. So our study of the $\mathrm{OEH}$ method will be carried out as if we were studying the pure initial value problem. We let $L_{h}$ be the finite difference replacement of the space operator $L$. Hence at the gridpoint $x_{j}$, where $j$ represents a multi-index $\left(j_{1}, \ldots, j_{d}\right), u_{t}=L u$ is replaced by the continuous time ordinary differential equation

$$
\dot{U}_{j}=L_{h} U_{j} \text {. }
$$

In what follows it is supposed that in each coordinate direction $L_{h}$ is based on second-order, three-point central differences on a uniform mesh. The restriction to uniform meshes in each coordinate direction is not essential. The OEH scheme allows a nonuniform mesh, but at most a three-point coupling in each coordinate direction. Hence one might also consider the use of simple one-sided spatial differencing. With regard to the convection-diffusion equation (1.1) we note that diffusion terms of the type $\nabla \cdot(\epsilon \cdot \nabla u), \epsilon$ a $d \times d$ matrix [9], are not allowed because of the cross-derivatives.

According to Gourlay [3-5] the OEH scheme for problem (2.1) is given by

$$
U_{j}^{n+1}=U_{j}^{n}+\tau \theta_{j}^{n} L_{h} U_{j}^{n}+\tau \theta_{j}^{n+1} L_{h} U_{j}^{n+1},
$$

where $\tau=t_{n+1}-t_{n}, U_{j}^{n}$ approximates $u$ at $\left(x_{j}, t_{n}\right)$ and

$$
\theta_{j}^{n}= \begin{cases}1 & \text { if }\left(n+\sum_{i} j_{i}\right) \text { is odd } \\ 0 & \text { if }\left(n+\sum_{i} j_{i}\right) \text { is even }\end{cases}
$$

If we take $n$ fixed and consider only the odd points, for this $n,(2.2)$ is just the forward Euler-central difference scheme. On the other hand, at the even points we recover the backward Euler-central difference scheme. Consequently, if we let $n$ fixed and first apply the forward 
scheme at all odd points and then the backward scheme at all remaining even points, we have carried out one step with the OEH scheme (2.2).

Due to the three-point coupling in each coordinate direction and the alternating use of forward and backward Euler methods, the process is only diagonally implicit. When applied to problem (1.1) it is even fully explicit (only division by scalars). This is also true for the nonlinear Burgers equation in divergence form

$$
u_{t}+a(u)=\epsilon \Delta u, \quad a(u)=\nabla \cdot(u ; u) .
$$

The convective form, $a(u)=(u \cdot \nabla) u$, requires that per gridpoint a $d \times d$ system of linear algebraic equations must be solved. Of course this is still very cheap.

By writing down two successive steps of scheme (2.3),

$$
\begin{aligned}
& U_{j}^{n+1}=U_{j}^{n}+\tau \theta_{j}^{n} L_{h} U_{j}^{n}+\tau \theta_{j}^{n+1} L_{h} U_{j}^{n+1}, \\
& U_{j}^{n+2}=U_{j}^{n+1}+\tau \theta_{j}^{n+1} L_{h} U_{j}^{n+1}+\tau \theta_{j}^{n+2} L_{h} U_{j}^{n+2},
\end{aligned}
$$

its connection to the Peaceman-Rachford method [3-5] is shown. In particular, if we let $h$ fixed, (2.5) may be interpreted as a second-order integration formula using stepsize $2 \tau$ for the ODE system defined by (2.1) (see also [10]). From formulation (2.5) one can also derive the attractive fast form [3-5] which halves the computational work of the complete step $n \rightarrow n+2$. A particularly advantageous feature is that this fast form can be implemented such that only one array of storage is required. It is evident that this may be of considerable interest for multi-dimensional problems. Finally, when applied to the problems (1.1), (2.4) the fast form implementation requires roughly the same number of operations per step as the forward Euler scheme. However, the $\mathrm{OEH}$ method has much better stability properties. We discuss this in the next section.

\section{Von Neumann stability for the linear convection-diffusion equation}

In this section we derive the critical time step for von Neumann stability of the OEH central difference scheme for the convection-diffusion equation (1.1). Let $h_{k}$ be the constant mesh width in the $k$ th direction and $H_{k}$ and $\delta_{k}^{2}$ the corresponding finite difference operators for the first and second derivative, respectively. Then $L_{h}$ can be written as

$$
L_{h} U_{j}=\sum_{k}\left(-\frac{q_{k}}{2 h_{k}} H_{k}+\frac{\epsilon}{h_{k}^{2}} \delta_{k}^{2}\right) U_{j}
$$

where the summation is from 1 to $d$.

The stability analysis exploits the equivalence to the leapfrog-DFF scheme. This equivalence emerges by eliminating variables at the time level $n+1$ in (2.5). For the odd points we then get the relation

$$
U_{j}^{n+2}=U_{j}^{n}+\tau L_{h} U_{j}^{n}+\tau L_{h} U_{j}^{n+2},
$$

and for the even ones

$$
U_{j}^{n+2}=2 U_{j}^{n+1}-U_{j}^{n} .
$$


By evaluating the linear expression $L_{h} U_{j}^{n+2}$ at the odd points and inserting (3.2) at the occurring even ones, relation (3.1) can be written as

$$
\left(1+\sum_{k} 2 \sigma_{k}\right) U_{j}^{n+2}=\left(1-\sum_{k} 2 \sigma_{k}\right) U_{j}^{n}-\sum_{k}\left(c_{k} H_{k}-4 \sigma_{k} \mu_{k}\right) U_{j}^{n+1},
$$

where $\mu_{k}$ is the standard averaging operator in the $k$ th direction and

$$
\sigma_{k}=\epsilon \tau / h_{k}^{2}, \quad c_{k}=q_{k} \tau / h_{k} .
$$

Scheme (3.3) is the combination of the leapfrog and DFF scheme (the case $d=1$ was studied earlier in [6]). Noteworthy is that (3.3) contains only grid values at the uncoupled set of odd-numbered points in space and time. Thus, if we ignore the start and completion of the $\mathrm{OEH}$ process and consider only the odd-numbered points, we may proceed with (3.3) for the investigation of linear stability. Note that $U_{j}^{n+1}$ in (3.2) is a grid value at an odd point. Hence if the computation at the uncoupled set of odd-numbered points is stable, we have also stability at all even points.

We shall now examine the stability of scheme (3.3). For this purpose we employ the classical method of von Neumann [12]. So we introduce the Fourier mode

$$
U_{j}^{n}=\xi^{n} \mathrm{e}^{\mathrm{i} \omega \cdot x}, \quad \omega=\left(\omega_{1}, \ldots, \omega_{d}\right)^{\mathrm{T}} \in \mathbb{R}^{d}, \quad \xi \in \mathbb{C}, \quad \mathrm{i}^{2}=-1,
$$

and substitute into (3.3) to give

$$
(1+\sigma) \xi^{2}+\left(\sum_{k} 2 c_{k} \text { i } \sin \theta_{k}-4 \sigma_{k} \cos \theta_{k}\right) \xi-(1-\sigma)=0,
$$

where $\theta_{k}=\omega_{k} h_{k}$ and $\sigma=2\left(\sigma_{1}+\cdots+\sigma_{d}\right)$. In what follows we demand von Neumann stability in the strict sense, that is

$$
|\xi| \leqslant 1, \quad \text { all }\left|\theta_{k}\right| \leqslant \pi
$$

Bearing in mind that $\sigma_{k}>0$ it then follows immediately from [11, Theorem 6.1] that we have stability iff the complex number $\lambda$ given by

$$
\lambda=\sum_{k} r_{k} \cos \theta_{k}-c_{k} \mathrm{i} \sin \theta_{k}, \quad r_{k}=2 \sigma_{k} / \sigma,
$$

satisfies $|\lambda| \leqslant 1$ for all $\left|\theta_{k}\right| \leqslant \pi$.

At this point we can make fruitful use of an interesting stability theorem due to Hindmarsh, Gresho and Griffiths [9] which they used in their stability analysis of the forward Euler-central difference scheme. As $\sum r_{k}=1, \lambda$ can be written as

$$
\lambda=1-\mathrm{i} \sum_{k} c_{k} \sin \theta_{k}+\sum_{k} r_{k}\left(\cos \theta_{k}-1\right)
$$

Their stability theorem then says that $|\lambda| \leqslant 1$ for all $\left|\theta_{k}\right| \leqslant \pi$ iff $\sum r_{k} \leqslant 1$ and

$$
\sum_{k} c_{k}^{2} / r_{k} \leqslant 1
$$

Hence we can conclude immediately that this condition is sufficient and necessary for von Neumann stability (in the strict sense) of scheme (3.3).

Equation (3.5) may be rewritten as

$$
\sum_{k} c_{k}^{2} / r_{k}=\sum_{k}\left(\frac{\tau}{h_{k}}\right)^{2} \sum_{k} q_{k}^{2} \leqslant 1,
$$


and observe that the diffusion parameter $\epsilon$ is absent in this condition. This is plausible because the DFF scheme is unconditionally stable for the pure diffusion problem $u_{t}=\epsilon \Delta u$. Next an interesting situation arises if we put $\epsilon=0$. Then scheme (3.3) reduces to the leapfrog scheme which is known to be stable in the strict sense of von Neumann iff the CFL condition holds:

$$
\sum_{k} \frac{\tau}{h_{k}}\left|q_{k}\right| \leqslant 1
$$

Consequently, from the Cauchy inequality

$$
\left(\sum_{k} \frac{\tau}{h_{k}}\left|q_{k}\right|\right)^{2} \leqslant \sum_{k}\left(\frac{\tau}{h_{k}}\right)^{2} \sum_{k} q_{k}^{2}
$$

it follows that the stability conditions are more restrictive for $\epsilon>0$ than for $\epsilon=0$. Hence if we add artificial diffusion to the $\mathrm{OEH}$ scheme for the pure convection problem we might destabilize the process. This observation is in clear contrast to the common practice which teaches us that introducing artificial diffusion has a stabilizing effect.

Observe that we have equality in (3.8) iff $h_{k}\left|q_{k}\right|$ is independent of $k$, so that only in this case the restrictions on $\tau$ and $h_{k}$ in (3.6), (3.7) are identical. Of course, this is trivially so for $d=1$ (see also [6]). If we put $h_{k}=h$, then (3.6), (3.7) lead to the time step restrictions

$$
\begin{aligned}
& \tau^{2} \leqslant h^{2} /\left(d \sum_{k} q_{k}^{2}\right), \\
& \tau^{2} \leqslant h^{2} /\left(\sum_{k}\left|q_{k}\right|\right)^{2} .
\end{aligned}
$$

We see that when one of the velocities $q_{k}$ dominates, the critical time step is approximately $\sqrt{d}$ times smaller than the critical time step imposed by the CFL condition.

We remark that the above pathological behaviour of the leapfrog-DFF scheme, and thus of the OEH scheme, has been observed earlier (see [13] and the references therein for numerical evidence). However, as far as we know, the expression for the critical time step implied by (3.6) is new.

Just for the sake of comparison we finally give the sufficient and necessary conditions for von Neumann stability of the forward Euler-central difference scheme for problem (1.1) [9]:

$$
\sum_{k} \frac{2 \epsilon \tau}{h_{k}^{2}} \leqslant 1, \quad \sum_{k} \frac{q_{k}^{2} \tau}{2 \epsilon} \leqslant 1 .
$$

The second of these is known as the convection-diffusion barrier. It shows that the forward Euler-central difference scheme becomes unconditionally unstable as $\epsilon \rightarrow 0$. In contrast, the $\mathrm{OEH}$ central difference scheme is stable for all $\epsilon \geqslant 0$ under condition (3.6).

\section{The DFF deficiency and global Richardson extrapolation}

This section is devoted to the second aspect of the OEH method considered in this paper, viz. the DFF accuracy deficiency by which we mean that convergence takes place for a smaller set of rules for refinement of the space-time mesh than allowed by the stability condition [12]. For 
example, if $\tau, h_{k} \rightarrow 0$ while satisfying (3.6) the solution of the leapfrog-DFF scheme (3.3) will converge to the solution of the problem

$$
u_{t}+(q \cdot \nabla) u=\epsilon \Delta u-\epsilon a u_{t t}, \quad a=\lim _{\tau, h_{k} \rightarrow 0} \sum_{k} \frac{\tau^{2}}{h_{k}^{2}} .
$$

Hence for convergence to (1.1) it is necessary that $\tau^{2}=\mathrm{o}\left(\left(\sum h_{k}^{-2}\right)^{-1}\right)$. Through the equivalence property the same conclusion is valid for the OEH scheme [2-5]. The equivalence to the leapfrog-DFF scheme cannot be derived for the nonlinear convection-diffusion equation. However, the DFF deficiency due to the viscous term $\epsilon \Delta u$ does still exist.

An immediate consequence of the DFF deficiency is that for a given space grid the OEH scheme may produce relatively inaccurate results unless $\tau$ is taken significantly smaller than necessary for stability. To a great extent, this disadvantage is compensated by the fact that per step the scheme is very cheap while on fixed space grids the fast form implementation (same costs as forward Euler) generates approximations which are second-order in $\tau$. For those applications where the disadvantage is still pertinent we suggest the employment of global Richardson extrapolation in time. The idea is to eliminate the term $\tau^{2} u_{t t}$ in (4.1). If we succeed in this elimination we have the usual convergence property because for stability $\tau$ must satisfy $\tau^{2}=\mathrm{O}\left(\left(\sum h_{k}^{-2}\right)^{-1}\right)$.

The basis for global Richardson extrapolation is formed by the existence of asymptotic expansions for the global error. Without attempting full rigor we shall briefly sketch this. For this purpose we shall instead of examining the leapfrog-DFF scheme for linear problems directly consider the OEH scheme (2.5), but on a fixed space grid. Hence we follow the ODE approach and interpret (2.5) as a one-step, second-order integration formula using stepsize $2 \tau\left(t_{n} \rightarrow t_{n+2}\right)$. The advantage is that we then do not need to distinguish between linear and nonlinear problems since the theory of asymptotic expansions for one-step methods for ODEs applies generally. Numerical evidence which shows that our ideas are correct will be provided in Section 5.

For convenience of presentation we introduce the operator notation

$$
U_{j}^{n+2}=S_{h}^{2 \tau} U_{j}^{n}, \quad n=0,2, \ldots,
$$

for the OEH scheme (2.5). Suppose that this scheme is applied from $t=0$ up to $t_{N}=N(2 \tau), N$ even, on a fixed spatial grid. Let

$$
\epsilon_{j}^{N}=U_{j}^{N}-U_{j}\left(t_{N}\right)
$$

be the global error at $t_{N}$ for the intermediate ODE problem (2.1). Now if we let $L_{h}$ be sufficiently smooth then it follows from numerical ODE theory $[7,8,15]$ that grid functions $e^{(2)}$, $e^{(3)}, \ldots$ exist independently of $\tau$ such that

$$
\epsilon_{j}^{N}=(2 \tau)^{2} e_{j}^{(2)}\left(t_{N}\right)+(2 \tau)^{3} e_{j}^{(3)}\left(t_{N}\right)+\cdots, \quad \tau \rightarrow 0 .
$$

The error term $(2 \tau)^{2} e_{j}^{(2)}\left(t_{N}\right)$ can be eliminated in the standard way by forming the combination

$$
\tilde{U}_{j}^{N}=\frac{1}{3}\left(4 U_{j}^{N}-V_{j}^{N}\right)
$$

where $U_{j}^{N}$ and $V_{j}^{N}$ are obtained from the two different integrations

$$
\begin{aligned}
& U_{j}^{n+2}=S_{h}^{2 \tau} U_{j}^{n}, \quad n=\mathrm{o}(2)(2 N-2), \\
& V_{j}^{0}=U_{j}^{0}, \quad V_{j}^{n+4}=S_{h}^{4 \tau} V_{j}^{n}, \quad n=\mathrm{o}(4)(2 N-4) .
\end{aligned}
$$


Consequently,

$$
\tilde{U}_{j}^{N}=U_{j}\left(t_{N}\right)-\frac{4}{3}(2 \tau)^{3} e_{j}^{(3)}\left(t_{N}\right)+\cdots .
$$

Obviously, the error functions $e^{(2)}, e^{(3)}, \ldots$ do depend on the grid spacing. In fact, due to the DFF deficiency they grow beyond bound if the grid spacing is refined. Of course, this growth is annihilated by the simultaneous reduction of $\tau$ which is necessary for stability. In connection with the DFF deficiency we now hypothesize that the lack of convergence is entirely due to the first term $(2 \tau)^{2} e_{j}^{(2)}\left(t_{N}\right)$ in (4.4). If this hypothesis is true the elimination of this term by global Richardson extrapolation trivially implies that when the grid is refined and $\tau$ is reduced according to the requirement of stability, that then $\tilde{U}_{j}^{N}$ will converge to $u\left(x_{j}, t_{N}\right)$.

In contrast to its ease of implementation, the $\mathrm{OEH}$ scheme (4.2) is not very amenable to error analysis so that we have not attempted to analyze the expression (4.4). Numerical experimentation with a nonlinear problem (see Section 5) indicates that the hypothesis is true and even more, namely that by the simple extrapolation device we get a truly second-order behaviour upon simultaneous reduction of the time and space mesh.

Global extrapolation as formulated in (4.6) is easy to implement. Having computed an approximation at $t=t_{N}$, one simply repeats the process but now with a double stepsize. This can be done in parallel or after completion of the first integration. The additional computational effort is $50 \%$ and an extra array of storage is required. Above we tacitly assumed that $\tau$ is constant. However, the process can also be carried out for variable stepsizes $[7,8,14-16]$ on the coarse grid without any additional difficulty. Finally we remark once more that a prerequisite for success of the extrapolation is that the asymptotics hold and thus that both integrations must be stable. Even marginal instability is not allowed because we then cannot count on a smooth global error. Lest we miss the obvious, the extrapolation makes no sense if the space error $U_{j}\left(t_{N}\right)-$ $u\left(x_{j}, t_{N}\right)$ dominates the time error (4.3).

\section{Numerical examples}

We have applied the OEH central difference scheme to two initial/boundary value problems of the first type for the inhomogeneous Burgers equation in divergence form (cf. (2.4)). We recall that for this type of equation the OEH central difference scheme is essentially explicit (only division by scalars). Because so far nothing has been mentioned on the treatment of time-dependent boundary values and inhomogeneous terms we first give the necessary details on the actual implementation we applied. Consider the semi-discrete approximation (cf. (2.1))

$$
\dot{U}_{j}=L_{h} U_{j}+F_{j}(t)
$$

where $F_{j}(t)$ is the contribution of the inhomogeneous term at the gridpoint $x_{j}$. It is supposed that the prescribed solution values on the boundary are contained in $L_{h} U_{j}(t)$ (at the relevant places).

While omitting the fast form modifications our odd-even hopscotch implementation is then based on the one-step scheme $\left(t_{n} \rightarrow t_{n+2}\right)$

$$
\begin{aligned}
& U_{j}^{n+1}=U_{j}^{n}+\tau \theta_{j}^{n} L_{h} U_{j}^{n}+\tau \theta_{j}^{n+1} L_{h} U_{j}^{n+1}+\tau F_{j}\left(t_{n}+\tau\right), \\
& U_{j}^{n+2}=U_{j}^{n+1}+\tau \theta_{j}^{n+1} L_{h} U_{j}^{n+1}+\tau \theta_{j}^{n+2} L_{h} U_{j}^{n+2}+\tau F_{j}\left(t_{n}+\tau\right),
\end{aligned}
$$


where it is of importance to notice that any prescribed solution value from the boundary occurring in $L_{h} U_{j}^{n}$ has been set at the stage $n$. This can be motivated by the observation that each of the two stages is consistent with (5.1). The inhomogeneous term is computed at $t_{n+1}$ in both stages in order to exploit fully the advantage of the fast form. When we apply the scheme only the points $t_{n}, t_{n+2}$ are used for output.
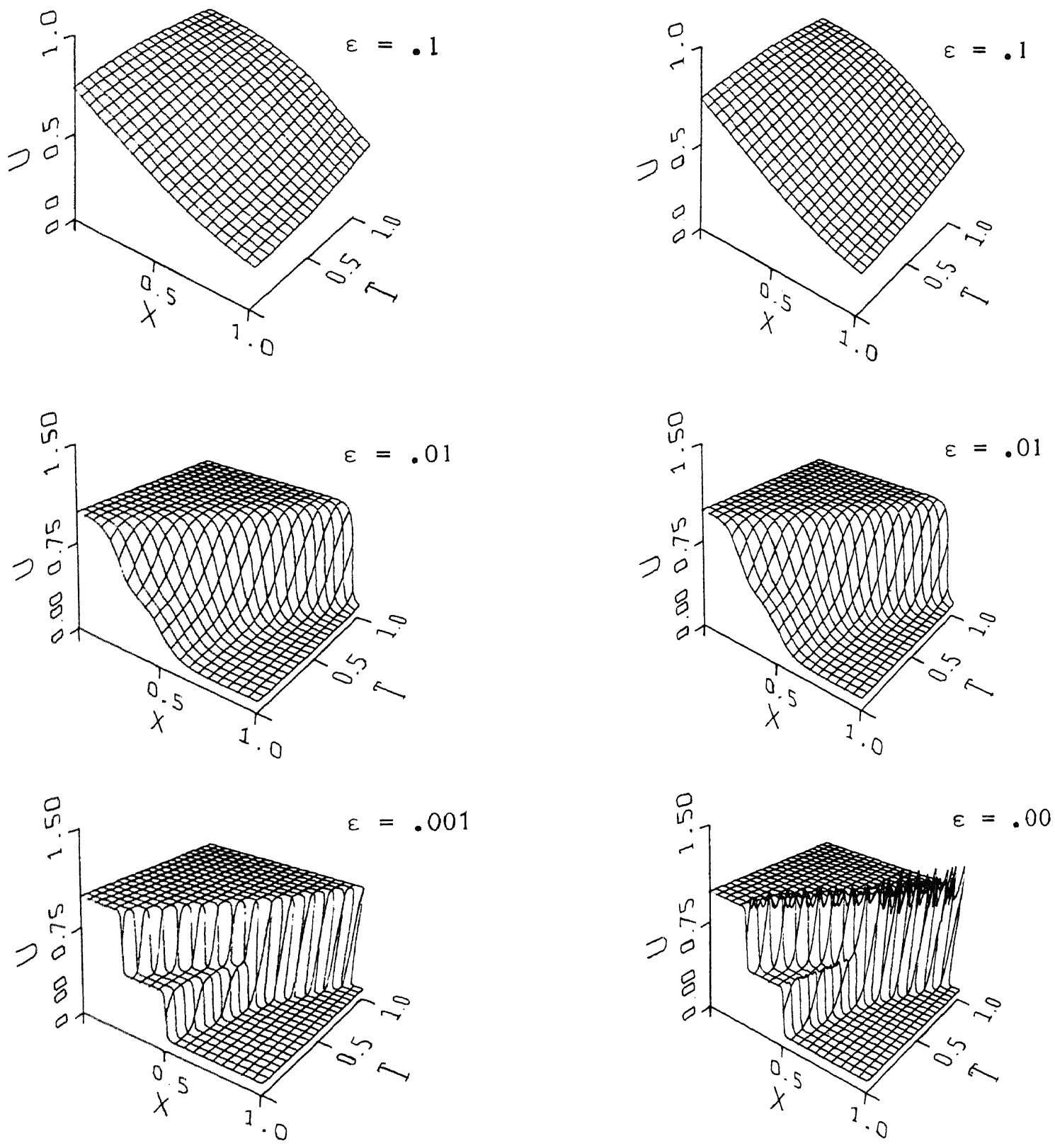

Fig. 1. Exact solutions (5.4) for three values of the

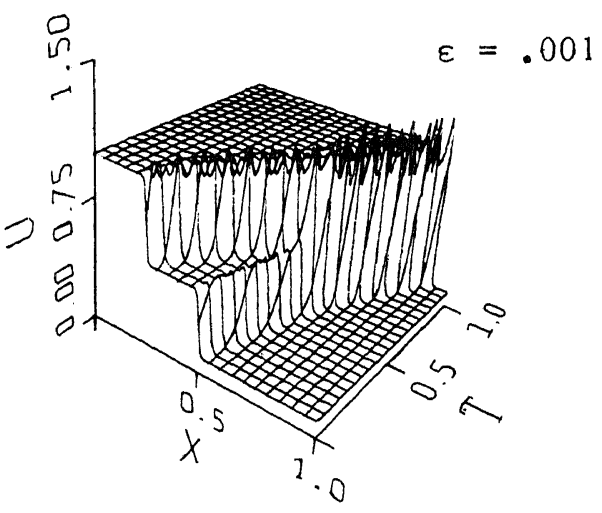
parameter $\epsilon$.

Fig. 2. Corresponding approximations generated by the $\mathrm{OEH}$ central difference scheme. We have used $h=\frac{1}{40}$, $\tau=\frac{1}{160}$ for $\epsilon=0.1$ and $h=\frac{1}{80}, \quad \tau=\frac{1}{320}$ for $\epsilon=0.01$, 0.001 . 
Table 1

Number of significant digits of the $\mathrm{OEH}$ scheme

\begin{tabular}{rlllll}
\hline$\tau^{-1}$ & $h^{-1}$ & & & & \\
\cline { 2 - 5 } & 20 & 40 & 80 & 160 & 320 \\
\hline 20 & 2.04 & & & & \\
40 & 2.66 & 2.03 & & & \\
80 & 3.24 & 2.63 & 2.03 & & \\
160 & 3.27 & 3.26 & 2.62 & 2.03 & \\
320 & 3.27 & 3.84 & 3.23 & 2.62 & 2.03 \\
640 & 3.26 & 3.88 & 3.86 & 3.23 & 2.62 \\
1280 & 3.26 & 3.87 & 4.45 & 3.84 & 3.22 \\
2560 & 3.26 & 3.87 & 4.48 & 4.47 & 3.83 \\
\hline
\end{tabular}

Table 2

Number of significant digits of the extrapolated $\mathrm{OEH}$ scheme

\begin{tabular}{|c|c|c|c|c|c|}
\hline \multirow[t]{2}{*}{$\overline{\tau^{-1}}$} & \multicolumn{5}{|l|}{$h^{-1}$} \\
\hline & 20 & 40 & 80 & 160 & 320 \\
\hline 40 & 3.19 & & & & \\
\hline 80 & 3.30 & 2.98 & & & \\
\hline 160 & 3.26 & 4.03 & 2.94 & & \\
\hline 320 & 3.26 & 3.87 & 4.66 & 2.93 & \\
\hline 640 & 3.26 & 3.86 & 4.50 & 4.35 & 2.93 \\
\hline 1280 & 3.26 & 3.86 & 4.47 & 5.21 & 4.29 \\
\hline 2560 & 3.26 & 3.86 & 4.47 & 5.08 & 5.97 \\
\hline
\end{tabular}

Problem 1. The one-space dimensional problem

$$
u_{t}+\frac{1}{2}\left(u^{2}\right)_{x}=\epsilon u_{x x}, \quad 0<t \leqslant 1, \quad 0<x<1,
$$

with the exact solution given by Whitham [17, Chapter 4]:

$$
u(x, t)=1-0.9 \frac{r_{1}}{r_{1}+r_{2}+r_{3}}-0.5 \frac{r_{2}}{r_{1}+r_{2}+r_{3}},
$$

where

$$
r_{1}=\exp \left(-\frac{x-0.5}{20 \epsilon}-\frac{99 t}{400 \epsilon}\right), \quad r_{2}=\exp \left(-\frac{x-0.5}{4 \epsilon}-\frac{3 t}{16 \epsilon}\right), \quad r_{3}=\exp \left(-\frac{x-\frac{3}{8}}{2 \epsilon}\right)
$$

We have integrated this problem for $\epsilon=0.1,0.01,0.001$. For these parameter values Fig. 1 and Fig. 2 show plots of the solution (5.4) and of corresponding numerical solutions, respectively. We see that when $\epsilon$ approaches zero the solution contains two shocks one of which is overtaken by the other. The plot for $\epsilon=0.001$ in Fig. 1 clearly shows this. Noteworthy is that the corresponding $\mathrm{OEH}$ central difference approximation reproduces this behaviour in a very acceptable way. Of course, the wiggles in the approximation are due to the difficulty of fitting the shock on a too coarse grid.

For $\epsilon=0.1$, Table 1 shows the minimum number of significant digits at $t=1, \min _{j}\left(-{ }_{10} \log (\right.$ abs. error at $\left.\left(x_{j}, 1\right)\right)$, for various values of $\tau$ and $h$. Note that since $\max (u(x, t))=1$ the critical time step for von Neumann stability is $\tau=h$. All computations with $\tau \leqslant h$ are indeed stable. Let us examine the results somewhat more closely. One then immediately recognizes the DFF deficiency: for a fixed mesh ratio $\tau / h$ the scheme fails to converge. Further, if we let $\tau \rightarrow 0$ and $h$ fixed and sufficiently small, one can observe the second-order behaviour of the OEH integration formula $\left({ }_{10} \log 4 \simeq 0.6\right)$. For $\epsilon=0.01,0.001$ these phenomena remain hidden due to too large errors in space. Also recall that the DFF deficiency originates entirely from the second-order term $\epsilon u_{x x}$.

For $\epsilon=0.1$ we present in Table 2 results of the extrapolated OEH scheme (4.5)-(4.6) (the meaning of the entries is the same as in Table 1). Let us examine the mesh ratio $\tau / h=\frac{1}{2}$. For this ratio the extrapolated scheme still suffers from the DFF deficiency, although the accuracy has improved. We think this is due to the fact that for the extrapolated scheme the mesh ratio $\tau / h=\frac{1}{2}$ is critical for von Neumann stability. Despite stability in such situations one may 
encounter a nonsmooth global error so that Richardson extrapolation cannot be of much use. For the mesh ratios $\tau / h=\frac{1}{8}, \frac{1}{16}, \ldots$, the extrapolated scheme appears to be free of the DFF deficiency. More precisely, for these ratios the extrapolated scheme behaves as a second-order scheme as we expected. For the ratio $\tau / h=\frac{1}{4}$ the extrapolation works for $\tau=\frac{1}{80}, \frac{1}{160}, \frac{1}{320}$, but then yields no further improvement. We again think that this is due to a nonsmooth global error caused by a very weak instability. For clarity, we recall that the DFF deficiency may become visible at the moment that the space error becomes smaller than the time error. Of course, only then the extrapolation in time can be fruitful. Our tables illustrate this clearly. We also emphasize that in all these cases the outcome of the extrapolation is positive, including those where the DFF deficiency is still visible due to a too large mesh ratio.

Problem 2. The inhomogeneous, two-space dimensional Burgers equation

$$
\begin{aligned}
& u_{t}+\left(u^{2}\right)_{x}+(u v)_{y}=\epsilon \Delta u+f_{1}(x, y, t), \quad 0<t \leqslant 1, \quad 0<x, y<1, \\
& v_{t}+(u v)_{x}+\left(v^{2}\right)_{y}=\epsilon \Delta v+f_{2}(x, y, t),
\end{aligned}
$$

where

$$
\begin{aligned}
& u(x, y, t)=2 \pi \sin (2 \pi x) \cos (y) z(x, y, t) \\
& v(x, y, t)=\cos (2 \pi x) \sin (y) z(x, y, t) \\
& z(x, y, t)=2 \epsilon \exp \left(-\left(4 \pi^{2}+1\right) \epsilon t\right) /\left(2+\exp \left(-\left(4 \pi^{2}+1\right) \epsilon t\right) \cos (2 \pi x) \cos (y) .\right.
\end{aligned}
$$

he functions $u, v$ constitute an exact solution of the homogeneous Burgers equation in convective form [1]. Note that this solution is purely artificial as both $u$ and $v$ vanish as $\epsilon \rightarrow 0$ or $t \rightarrow \infty$. However, it is acceptable for our numerical illustration purpose which concerns the DFF deficiency and the Richardson extrapolation procedure. In Tables 3 and 4 we present results of the $\mathrm{OEH}$ central difference scheme $\left(h_{1}=h_{2}=h\right)$ and of the extrapolation, respectively, for $\epsilon=0.02$ and $t=1$. The results concern the $u$-component. The numbers in the tables have the same meaning as in Table 1.

Noteworthy is that in Table 3 the DFF deficiency again manifests itself very clearly. On the other hand, the extrapolated OEH scheme turns out to be free of this deficiency as is shown in Table 4. It nicely shows the expected second-order behaviour, in particular for the mesh ratio $\tau / h=\frac{1}{4}$.

\begin{tabular}{|c|c|c|c|c|}
\hline \multirow{2}{*}{$\overline{\tau^{-1}}$} & \multicolumn{4}{|l|}{$h^{-1}$} \\
\hline & 10 & 20 & 40 & 80 \\
\hline 20 & 2.71 & 2.78 & & \\
\hline 40 & 2.66 & 3.45 & 2.73 & \\
\hline 80 & & 3.32 & 3.39 & 2.71 \\
\hline 160 & & & 4.06 & 3.34 \\
\hline 320 & & & & 4.00 \\
\hline
\end{tabular}

Our experience with the two numerical examples justifies the conclusion that the extrapolated $\mathrm{OEH}$ scheme possesses the normal convergence property. A very attractive feature of the global

Table 3

Number of significant digits of the $\mathrm{OEH}$ scheme
Table 4

Number of significant digits of the extrapolated $\mathrm{OEH}$ scheme

\begin{tabular}{rllll}
\hline$\tau^{-1}$ & $h^{-1}$ & & & \\
\cline { 2 - 5 } & 10 & 20 & 40 & 80 \\
\hline 40 & 2.64 & 3.22 & & \\
80 & & 3.23 & 3.80 & \\
160 & & & 3.83 & 4.30 \\
320 & & & & 4.43 \\
\hline
\end{tabular}


extrapolation in time is its simplicity. This classique technique can be placed on top of any OEH implementation, even for variable time steps. This allows the possibility of using it for stepsize control purposes, e.g. to avoid instabilities. In this section we have concentrated on convectiondiffusion problems. The technique may also prove useful for pure diffusion problems. For such problems the OEH scheme has better stability properties so that the need for more accuracy in time will exist more frequently. Finally, we stress once more that the extrapolation idea assumes that the asymptotics hold. A prerequisite is thus that on both grids in time we have stability. In this respect, pure diffusion problems are even more attractive for application of the OEH extrapolation scheme.

\section{References}

[1] C.A.J. Fletcher, A comparison of finite element and finite difference solutions of the one- and two-dimensional Burgers equation, J. Comput. Phys. 51 (1983) 159-188.

[2] P. Gordon, Nonsymmetric difference equations, SIAM J. Appl. Math. 13 (1965) 667-673.

[3] A.R. Gourlay, Hopscotch: a fast second order partial differential equation solver, J. Inst. Math. Appl. 6 (1970) 375 390.

[4] A.R. Gourlay, Some recent methods for the numerical solution of time-dependent partial differential equations, Proc. Roy. Soc. London A 323 (1971) 219-235.

[5] A.R. Gourlay, Splitting methods for time dependent partial differential equations, in: D. Jacobs, ed., The State of the Art in Numerical Analysis (Academic Press, London/New York/San Francisco, 1977) 757-791.

[6] A.R. (jourlay and J.LI. Morris, Hopscotch difference methods for nonlinear hyperbolic systems, IBM J. Res. Develop. Io (1972) 349-353.

[7] E. Hairer and C. Lubich, Asymptotic expansions of the global error of fixed-stepsize methods, Numer. Math. 45 (1984) $345-360$.

[8] P. Henrici, Discrete Variable Methods in Ordinary Differential Equations (Wiley, New York/London, 1962).

[9] A.C. Hindmarsh, P.M. Gresho and D.F. Griffiths, The stability of explicit Euler time-integration for certain finite-difference approximations of the multi-dimensional advection-diffusion equation, Internat. J. Numer. Meth. Fluids 4 (1984) 853-897.

[10] P.J. van der Houwen and J.G. Verwer, One-step splitting methods for semi-discrete parabolic equations, Computing 22 (1979) $291-309$.

[11] J.J.H. Miller, On the location of zeros of certain classes of polynomials with applications to numerical analysis, $J$. Inst. Maths. Appl. 8 (1971) 397-406.

[12] R.D. Richtmyer and K.W. Morton, Difference Methods for Initial-Value Problems (Interscience, New York/ London/Sydney, 1967).

(13) U. Schumann, Linear stability of finite difference equations for three dimensional flow problems, J. Comput. Phys. 18 (1975) $465-470$.

[14] L.F. Shampine and H.A. Watts, Global error estimation for ordinary differential equations, ACM Trans. Math. Software 2 (1976) $172-186$.

[15] H.J. Stetter, Analysis of Discretization Methods for Ordinary Differential Equations (Springer, Berlin/Heidelberg/ New York, 1973).

[16] J.G. Verwer and H.B. de Vries, Global extrapolation of a first order splitting method, SIAM J. Sci. Statist. Comp. o (3) (1985).

[17] G.B. Whitham, Linear and Nonlinear Waves (Wiley-Interscience, New York, 1974). 